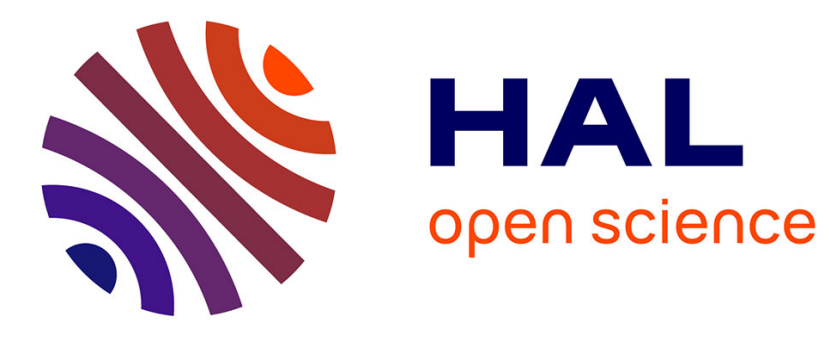

\title{
Product and Process Configuration: A constraint based approach
}

\author{
Michel Aldanondo, Élise Vareilles, Meriem Djefel, C. Baron
}

\section{To cite this version:}

Michel Aldanondo, Élise Vareilles, Meriem Djefel, C. Baron. Product and Process Configuration: A constraint based approach. IEEM: 2008 INTERNATIONAL CONFERENCE ON INDUSTRIAL ENGINEERING AND ENGINEERING MANAGEMENT, 2008, Singapore, Singapore. p. 2010-2014, 10.1109/IEEM.2008.4738224 . hal-01599443

\section{HAL Id: hal-01599443 \\ https://hal.science/hal-01599443}

Submitted on 17 Oct 2017

HAL is a multi-disciplinary open access archive for the deposit and dissemination of scientific research documents, whether they are published or not. The documents may come from teaching and research institutions in France or abroad, or from public or private research centers.
L'archive ouverte pluridisciplinaire HAL, est destinée au dépôt et à la diffusion de documents scientifiques de niveau recherche, publiés ou non, émanant des établissements d'enseignement et de recherche français ou étrangers, des laboratoires publics ou privés. 


\title{
Product and Process Configuration: A constraint based approach
}

\author{
M.Aldanondo ${ }^{1}$, E.Vareilles ${ }^{1}$, M.Djefel ${ }^{1,2}$ C. Baron $^{2}$ \\ ${ }^{1}$ University of Toulouse - Mines Albi - CGI, Albi, France \\ ${ }^{2}$ University of Toulouse - LATTIS - INSA, Toulouse, France
}

\begin{abstract}
This communication deals with the possibility of coupling together interactive product configuration tools with process planning tools in order to communicate and make consistent decisions made from one side to the other side. The first section introduces the problem and the general ideas of the proposed solution. Then, two constraints based models, respectively relevant to product configuration and process planning, are presented. Finally first investigations for coupling these two models and associated problems are discussed. An example illustrates our proposal through out the paper.
\end{abstract}

Keywords - Product, Process, Configuration, Constraint

\section{INTRODUCTION}

The aim of this communication is to present the first results of a study dealing with the development of an aiding system that will simultaneously allow interactive configuration of a product and interactive planning of its production process. It seems rather logical that on the one hand, product configuration decisions have strong consequences on the planning of its production process and that, on the other hand, planning decisions provide hard constraints to product configuration. Therefore, we propose to associate these two problems in order to allow (i) the propagation of the consequences of each product configuration decision toward the planning of its production process and (ii) the propagation of the consequences of each process planning decision towards the product configuration. This should reduce or avoid planning impossibilities due to product configuration and configuration impossibilities due to production planning.

As far as we know, this kind of problem has not been addressed yet. In the design community, for more than fifteen years, many works around Axiomatic Design (Suh in 1990 [1]), Design Structure Matrix (Steward in 1981 [2]), or Function Behaviour Structure (Gero in 1990 [3]), have proposed different domains (customer, functions, requirements, behaviour, physical, process, resource...) in order to characterize product development. A recent paper from the DSM community [4] proposed a mapping of the four domains: functions, components, process and resources (figure 1). Our studies are based on these four domains: we associate (i) the function and component views within product configuration and (ii) the process and resource views within the planning of the production process.

In the configuration community, many authors (among them Sabin [5] or Soininen [6]) have shown that product configuration could be efficiently modelled and aided when considered as a Constraints Satisfaction Problem (CSP). In a same way, authors interested in scheduling (such Dechter [7] or Laborie [8]) have shown that project planning could be also modelled and aided when considered as a CSP. A CSP is a triplet $\{\mathrm{X}, \mathrm{D}, \mathrm{C}\}$ where $\mathrm{X}$ is a set of variables, $\mathrm{D}$ a set of finite domains (one for each variable) and $\mathrm{C}$ a set of constraints linking the variables [9]. The variables can be either discrete or continuous. The constraints define the possible or forbidden combinations of values for a set of variables. Once given these elements, we propose to consider configuration and planning problems as two constraint satisfaction problems. In order to propagate decision consequences between the two problems, we suggest to link these two constraint based models.

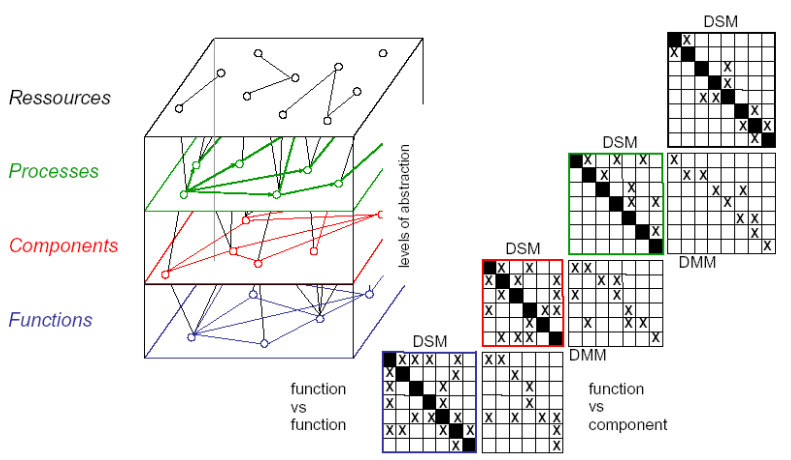

Fig. 1. Four domains - from the paper (Lindemann U., 2007 [4])

The communication is therefore organized as follow: sections 2 and 3 address product configuration and planning issues. The association of the two constraints models is then investigated and discussed in section 4 . A detailed example runs through out the paper.

\section{PRODUCT CONFIGURATION}

This section first defines the configuration problem then the constraint model with propagation techniques, before concluding with the description of the example.

\section{A. Configuration Problem Description.}

From previous works ([6] or [10]), it seems that some common features defining configuration could be:

- hypothesis: a product is a set of components,

- given: a generic model of a configurable product able to represent a family of products with all possible variants and options, that gathers (1) a set of component groups (2) a set of product properties and 
(3) a set of various constraints that restrict possible combinations of components and property values;

- given a set of customer requirements, in which each requirement can be expressed by a selection (or a domain restriction) of a component or a property value,

- configuring can be defined as "finding at least one component set that satisfies all the constraints and the customer requirements".

We associate previous properties with some kind of product description that matches the "functions" domain while component and component groups are associated with the physical or" components" domains of section 1 .

\section{B. Constraint Model and Propagation Techniques}

Each group of components and each product property is associated with a configuration variable defined on a symbolic domain. Each component and each property value corresponds with one value of the variable. The constraints represent the allowed or excluded (solid or dot lines of figure 2) combinations of components and property values. The Dynamic extension of the CSP, DCSP proposed by [11], is used in order to control the variable existence with activity constraints (arrows on figure 2: a specified value of a variable " $\mathrm{X}$ " implies the existence of variable "Y"). As we target interactive assistance, constraint propagation is obtained thanks to filtering algorithms relying on arc consistency techniques, defined for discrete CSP and DCSP.

\section{Example relevant to Product Configuration.}

The Figure 2 shows the configuration model of our example. The considered product is a very simple crane. In its functional (or descriptive) view (left part of Figure $2)$, the product can be defined with:

- four configuration variables: (1) V length, the height of the crane (two possible values: $4 \mathrm{~m}$ and $8 \mathrm{~m}$ ), (2) $\mathrm{H}$ length, the width of the crane (two values: $2 \mathrm{~m}$ and $4 \mathrm{~m}$ ), (3) $\mathrm{M}$ load, the maximum load (two values less than one ton between 1 and 2 tons, (4) $\mathrm{Ctr}-\mathrm{Cab}$, modulating the existence of a control cabin with two values "yes" and "no".

- three constraints that reduce the solution space and exclude combinations of values: (1) $(\mathrm{V}$ length $=$ " $4 \mathrm{~m}$ ") incompatible with (H_length $=$ " $4 \mathrm{~m}$ ") a crane cannot have the same height and width, (2) (M load = " $1 \mathrm{t}<<2 \mathrm{t}$ ") incompatible with (H_length = " $4 \mathrm{~m}$ ") the maximum load is not compatible with the larger width, (3) (V_length = "4m") incompatible with (Ctr$\mathrm{Cab}=$ "yes") a height of four meters forbids the presence of a control cabin.

In its physical (or component) view (right part of Figure

2) the model shows:

- four groups of components: (1) V_Struct, the vertical structure gathering four physical components combining different acceptable loads and lengths:
"V4_n", "V4_s", "V8_n", "V8_s", (2) H_Struct, the horizontal structure gathering two physical components according to its length "H2_n", "H4_n", (3) Engine, the crane engine gathering 2 physical components with two different power "E_lp", "E_hp", (4) Cab, the control cabin gathering two physical components according to the engine power "Cab_lp", "Cab_hp".

- in this physical view, a single constraint associates the possible engine and the control cabin (solid line between variable values): (Engine="E_lp") compatible ( $\mathrm{Cab}=$ "Cab_lp") and (Engine="E_hp") compatible ( $\mathrm{Cab}=$ "Cab_hp").

The functional and physical views are linked with constraints that show how the components can fulfil the parameters of the functional view. Three constraints show the following possible combinations: (1) the height of the crane (V length) and the maximum load (M load) are linked with the vertical structure (V_Struct), (2) the maximum load (M_load) impacts the engine selection (Engine), (3) the width of the crane (H_length) is associated with the horizontal structure (H Struct). One activity constraint is necessary to allow triggering the existence of the variable $(\mathrm{Cab})$ corresponding with the component group "cab".

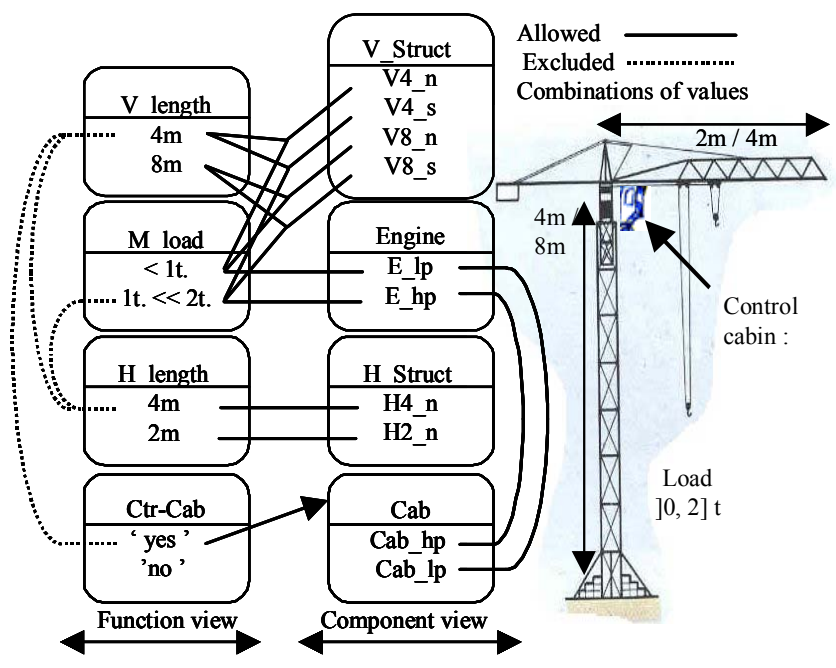

Fig. 2. Product configuration model

\section{PRODUCTION PLANNING}

This section first defines the planning problem then describes the constraint model with relevant propagation techniques, before illustrating them on the example.

\section{A. Planning Problem Description}

As we address production planning, we will consider that the production process is a set of task entities. At the present time we only consider planning with infinite resource capacity. A task entity is defined with:

- temporal parameters: possible start time (pst), possible finishing time (pft), possible duration (pdt), 
- $\quad$ resource parameters: required resource (rrs), quantity of required resource (qrs).

- compatibility constraints can link possible duration (pdt), with required resource (rrs) and/or quantity of required resource (qrs).

The three temporal parameters are numerically defined with intervals while resource parameters can remain symbolic (as we consider infinite resource capacity). The production process is defined with a set of task and a set of precedence constraints between task expressing that task $\mathrm{Y}$ is after task $\mathrm{X}$ or: Y.pst $>\mathrm{X}$.pft These kinds of constraints have certain similarities to the Allen's primitives [12] (before, after, starts, finishes...).

With these elements the production process can gather sequential tasks and parallel tasks as shown with the "AND" node (\&) in the left part of figure 3. Planning decisions can correspond with a temporal parameters value selection or domain restriction, a resource and/or resource quantity selection or domain restriction. In order to be able to select a path or a branch in the process (OR node in the right part of figure 3 ) it is necessary to be able to control the existence of the task entities.

Interactive planning corresponds with the propagation of all the constraints in order to reduce the definition domain of all temporal parameters, resource parameters and to select the path in the case of "XOR" nodes.

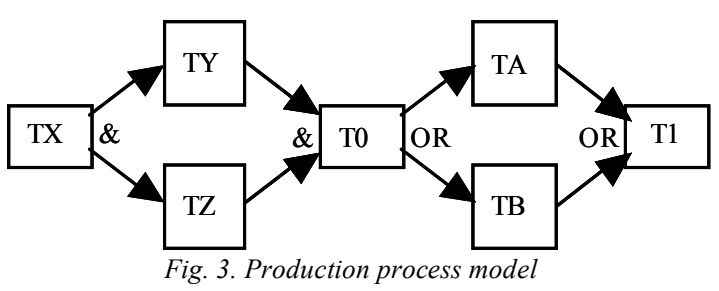

\section{B. Constraint Model and Propagation Techniques}

A task entity (TI, for task "I") is created and gathers three real variables defined with intervals corresponding with the three temporal parameters:

- $\quad$ pst: possible start time (TI.pst),

- pdt: possible duration (TI.pdt),

- $\quad$ pft: possible finishing time (TI.pft).

When all the variables are numerical and each constraint written with a mathematical formula, $\mathrm{f}\left(\mathrm{x}_{1}, \mathrm{x}_{2} \ldots \mathrm{x}_{\mathrm{n}}\right)(=><)$ 0 , bound consistency, proposed by [13] and based on interval arithmetic [14], proposes filtering techniques that operate fine if the following conditions are fulfilled:

- $\mathrm{f}\left(\mathrm{x}_{1}, \mathrm{x}_{2} \ldots \mathrm{x}_{\mathrm{n}}\right)=0$ can be projected on any variable $\mathrm{x}_{\mathrm{i}}$ meaning that a function $\mathrm{f}_{\mathrm{i}}$ exists as: $\mathrm{x}_{\mathrm{i}}=\mathrm{f}_{\mathrm{i}}\left(\mathrm{x}_{1}, \mathrm{x}_{2} \ldots . \mathrm{x}_{\mathrm{i}-}\right.$ $\left.1, \mathrm{x}_{\mathrm{i}+1}, \ldots \mathrm{x}_{\mathrm{n}}\right)$,

- $\quad$ any projections $\mathrm{f}_{\mathrm{i}}$ is continuous and monotonous.

As we consider only the two following kinds of constraints:

- $\quad$ TY.pst $>$ TX.fst, expressing that task $\mathrm{Y}$ is after task $\mathrm{X}$
- $\quad$ TI.pft $=$ TI.pst + TI.pdt , expressing the relation between starting, finishing times and duration,

we can use bound consistency filtering algorithm with the various projections of the two previous kinds of constraints. Planning works perfectly as far as there is no "XOR" node.

In order to deal with the "XOR" node we propose to define a meta-task entity "XOR" that gathers two tasks that are linked with a "XOR" node. Assume that we have a process $\mathrm{T} 0$ then $\mathrm{TA}$ or $\mathrm{TB}$ then $\mathrm{T} 1$ (as in the right part of figure 3 ):

- TA with TA.pst, TA.pdt, TA.pft

- $\quad$ TB with TB.pst, TB.pdt, TB.pft

The possible starting and finishing times of the XOR AB meta-task gathering TA and TB, XOR_AB.pst and XOR_AB.pft, are constrained by:

- XOR_AB.pst $>$ T0.pft meta-task starts after T0

- $\quad$ XOR_AB.pst $\leq$ TA.pst meta-task starts before TA

- XOR_AB.pst $\leq$ TB.pst meta-task starts before TB

- $\quad$ TA.pft $\leq$ XOR_AB.pft meta-task finishes after TA

- $\quad$ TB.pft $\leq$ XOR_AB.pft meta-task finishes after TB

- T1.pst $>$ XOR_AB_.pft meta-task finishes before T1

The possible duration of the XOR_AB meta-task is constrained by:

- XOR_AB.pdt = TA.pdt U TB.pdt, the possible duration of the meta-task equals the union of the possible duration of tasks $\mathrm{A}$ and $\mathrm{B}$

- XOR_AB.pdt $>0$, the possible duration of the metatask is always strictly positive.

The meta-task "XOR" temporal parameters can be therefore propagated with the same bound consistencyfiltering algorithm.

\section{Example relevant to Production Planning.}

The Figure 4 shows the planning model relevant to the production of the crane example. Only possible duration (pst) for each task is provided. A required resource with a quantity is defined just for two tasks (Manuf Structure and Deliver). A launching task has been added. This gives:

- $\quad$ Launch (L): event that allows to set a launching time, L.pst $=$ L.pdt $=$ L.pft $=0$.

- Manuf structure (MS) corresponds with the manufacturing of the two structures (V Struct and H_Struct), a small and a large machine can be used with a quantity 1 , MS.pdt $=[3,6]$, MS.rrs = \{large_mach, small_mach $\},$ MS.qrs $=$ "1".

- Source Engine (SE): corresponds with the sourcing of the engine (E_lp and E_hp), SE.pdt $=[2,4]$.

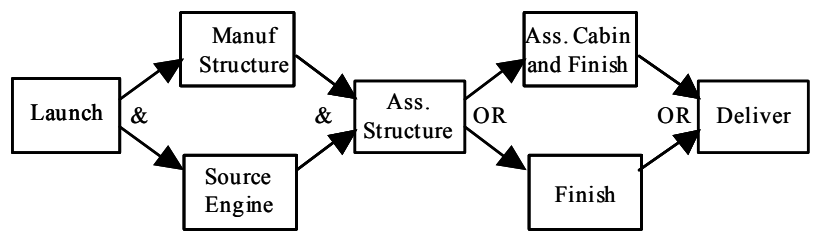

Fig. 4. Production process model of the crane. 
- Ass Structure (AS): assembly of the structures with the engine, duration always equal to 2, AS.pdt $=2$.

- Ass Cabin and finish (ACF): if a cabin is present in the configuration, ACF.pdt $=\{0,[3,4]\}$.

- Finish (F): if no cabin in the configuration, F.pdt $=$ $\{0,[1,2]\}$

- $\quad$ XOR_ACFF.pdt $=$ ACF.pdt U F.pdt, and $>0$, XOR_ACFF.pdt $=\{[1,2],[3,4]\}$.

- Deliver (D): corresponds with the delivering of the completed crane. Two different transportation resources can be used and modulate duration, D.pdt $=[1,2]$, D.rrs $=\{$ fast_trans,slow_trans $\}$, D.qrs $=$ "1" . Constraint (D.rrs , D.pdt): allowed combinations: $\{($ slow_trans, $[1.5,2]),($ fast_trans, $[1,1.5])\}$

Figure 5 shows previous elements after constraint propagation when starting and finishing dates have an initial interval value $[0,20]$.

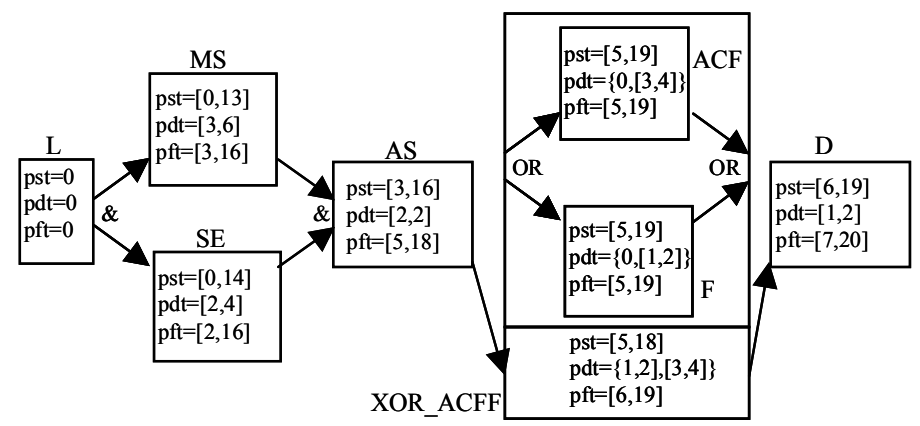

Fig. 5. Production process after first propagation.

\section{COUPLING PRODUCT AND PLANNING}

\section{A. Coupling Constraints}

A coupling constraint is a compatibility constraint that links a variable of the configuration model with a variable of the planning model. Any variable of the configuration model can belong to a coupling a constraint. On the planning model side, three cases can be identified:

- the planning variable is a resource parameter (rrs or qrs). This allows to propagate the impact of a configuration decision on the selection of the required resource and/or resource quantity, reverse behaviour from resource selection to product configuration is also possible,

- the planning variable is the temporal parameter duration (pst) that does not belong to a XOR metatask. This allows to propagate the impact of a configuration decision on the modulation of the duration of a task, reverse behaviour from duration modulation to product configuration is also possible,

- when the temporal parameter duration (pst) belong to a XOR meta-task, the previous behaviour is completed with the possibility to select one of the task of the meta-task.

\section{B. Illustration with the Crane Example}

The three previous cases are illustrated as follow:

- the planning variable is a resource parameter:

The resource of the task manufacturing structure, MS.rrs, is linked with the height of the crane, V_Length, allowed combinations: (V_Length, MS.rrs): $\{(4 \mathrm{~m}$, small_mach $),(8 \mathrm{~m}$, large_mach $)\}$

- the planning variable is a duration parameter that does not belong to a XOR meta-task, two cases:

(1) The duration of the task manufacturing structure, MS.pdt, is linked with the maximum load, M_load, allowed combinations: (M_load, MS.pdt):

$\{(<1 \mathrm{t},[3,4.5]),(1 \mathrm{t}<<2 \mathrm{t},[4.5,6])\}$.

(2) The duration of the task source engine, SE.pdt, is linked with the engine component, Engine, allowed combinations: (Engine, SE.pdt):

$\{$ ("E_lp", [2, 3]), (“E_hp", [3, 4]) \}.

- the planning variable is a duration parameter that belong to a XOR meta-task in order to select the existence of a task:

The duration of the task assemble cabin and finish, ACF.pdt, is linked with the existence of a control cabin, Ctr-Cab, with the following allowed combinations: Constraint (Ctr-Cab, ACF.pdt):

$\{$ ("no", [0]), (“yes", $[3,4])\}$.

\section{Propagating product decisions towards planning.}

If we assume the following configuration decisions: $\mathrm{V}$ length $=$ " $8 \mathrm{~m}$ ", H_length $=$ " $2 \mathrm{~m}$ ", M_load $=$ " $1 \mathrm{t}<<2 \mathrm{t}$ ", and leave open the decision relevant to the existence of a control cabin. The configuration provide the component set: V_Struct $=$ "V8_n", H_Struct $=$ "H2_n", Engine $=$ "E_hp". Then, coupling constraints provide:

- the selection of resource: MS.rrs = "large_mach"

- the duration of the tasks: MS.pdt $=[4.5,6]$ and SE.pdt $=[3,4]$

- $\quad$ finishing and stating times of figure 6 .

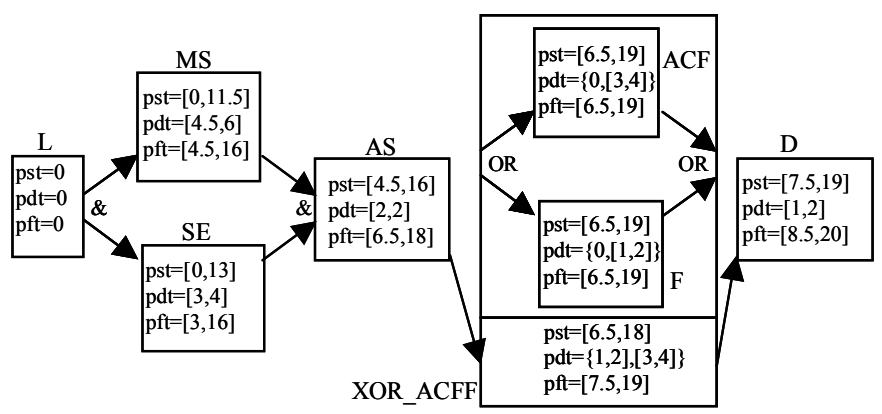

Fig. 6. Planning after product configuration

\section{Propagating planning decisions towards configuration.}

One of the consequences of the coupling provides an increase of the total finishing time (D.pft) from [7, 20] to $[8.5,20]$. If we assume now that the person in charge of planning wants to secure the manufacturing tasks and 
selects the maximum duration for them: MS.pft $=[6,6]$ and SE.pft $=[4,4]$. Planning propagation gives the planning of figure 7 with a total finishing time D.pft $=$ $[10,20]$.

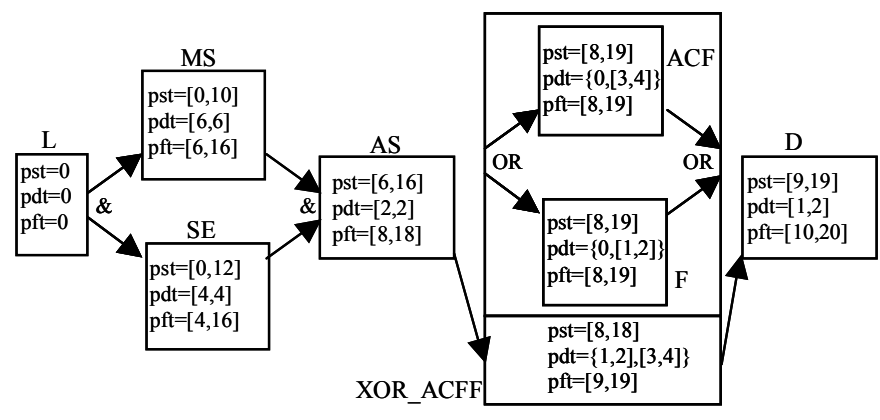

Fig. 7. Planning after manufacturing freezing duration

If we assume that the total finishing time D.pft should be now less than 11. This implies:

For task D:

- $\quad$ D.pft $=[10,11] \quad$ and D.pst $=[9,10]$,

For meta-task XOR_ACFF, task F and ACF:

- $\quad X O R \_A C F F . p f t=[9,10]$ and XOR_ACFF.pst $=[8,9]$

- $\quad$ F.pft $=[8,10] \quad$ and F.pst $=[8,10]$

- $\quad$ ACF.pft $=[8,10]$ and ACF.pst $=[8,10]$

and the propagation of the constraints expressing that finish time equal start time plus duration $(\mathrm{Tpft}=\mathrm{T}$.pst + Tpdt ) and the constraint of the XOR node gives for :

- $\quad$ Task ACF: ACF.pdt $=[0,0]$

- $\quad$ Task F: F.pdt = $[1,2]$

- $\quad$ meta-task XOR_ACFF: XOR_ACFF.pdt $=[1,2]$

Planning propagation has therefore selected task $\mathrm{F}$ and cancelled task ACF. The null value for ACF.pdt is then propagated to the configuration model and forbid the selection of a control cabin by reducing the variable CtrCab to the value "no". A planning decision has therefore provided a restriction on configuration possibilities.

\section{CONCLUSION}

The aim of this communication was to present the first results and prospective ideas about the development of an interactive aiding system that simultaneously allows product configuration and production planning. The main interest and goal of this system is to be able to take into account: product configuration decisions when dealing with production planning and production planning decisions when dealing with product configuration.

We have first presented our problem and proposed to associate each problem with a constraint satisfaction problem and to link the two problems with coupling constraints. Then, a simple configuration problem was defined and modelled with two views: functions and components. Constraint propagation was achieved thanks to arc consistency techniques. An example dealing with a crane was described. The planning problem was defined and modelled thanks to a network of tasks that allows
"AND" and "OR" nodes. Bound consistency techniques were used to propagate constraints. The crane example was extended with production planning entities. The final section described the constraints that can associate the two previous models in order to pass decisions made from one to the other. The crane example was used to show (i) how configuration decisions were propagated to planning and (ii) how planning decisions were propagated to configuration.

One of the interests interest of the proposed system is that it only relies on the simple assembly of two constraint filtering techniques: arc consistency for discrete CSP and bound consistency for numerical CSP, The other main interest is relevant to the application domain, where the possibility to manage interactions between product configuration and production planning has been addressed. These elements must be considered as primary results that need to be consolidated with structured (or multi-level) planning, finite resource capacity planning and less routine design. An other important issue will be also to provide a method for constraint model designing and CSP consistency checking.

\section{ACKNOWLEDGMENT}

The authors would like to thank:

- the French National Research Agency (ANR) and the Aerospace Valley for their involvement,

- their partners in the ATLAS project: Anyware Technologies, Pulsar Innovations, Sigma Plus and LAAS-CNRS from Toulouse, LGP-ENIT from Tarbes, and IMS-LAPS from Bordeaux - France.

\section{REFERENCES}

[1] N. Suh, "The principes of design", Oxford Series 1990.

[2] D. Steward, "The Design Structure System: A Method for Managing the Design of Complex Systems" IEEE Transactions on Engineering Management, vol. 28, pp. 71-74, 1981.

[3] J.S Gero, "Design prototypes: a knowledge representation schema for design", AI magazine, Vol $11 \mathrm{n}^{\circ} 4$, p 26-36 1990.

[4] U. Lindemann, "A vision to overcome "chaotic" design for X processes in early phases", in Proc. Of ICED, Paris, France, 2007.

[5] D. Sabin, R.Weigel, "Product Configuration Frameworks - A survey", IEEE Intelligent Systems, vol. 13, n 4, 1998, p. 42-49.

[6] T. Soininen, T.Tiihonen, T. Männistö, R. Sulonen, "Towards a General Ontology of Configuration”, AIEDAM, vol. 12, n 4, 1998

[7] R. Dechter, I. Meiri, J. Pearl, "Temporal Constraint Satisfaction Problems", Artificial Intelligence, 49, pp. 61-95, 1991.

[8] P. Laborie. "Algorithms for Propagating Resource Constraints in AI Planning and Scheduling: Existing Approaches and New Results". Artificial Intelligence, 143, pp. 151-188. 2003.

[9] E. Tsang, "Foundations of constraints satisfaction", Academic Press, London, 1993.

[10] M.Aldanondo, K. Hadj-Hamou, G. Moynard, J. Lamothe, "Mass customization and configuration: Requirement analysis and constraint based modeling propositions.”, ICAE IOS Press, Vol. $10, \mathrm{n}^{\circ} 2, \mathrm{pp} 177-189,2003$

[11] S. Mittal , B. Falkenhainer, "Dynamic Constraint Satisfaction Problems", Proc.s of the $9^{\text {th }}$ AAAI, Boston, USA, 1990, p. 25-32.

[12] J. Allen, "Maintening knowledge about temporal intervals", Communication of the CACM, vol 26(11), pp. 832-843, 1983.

[13] O. Lhomme - "Consistency techniques for numerical CSPs" - proc of IJCAI 93, Chambéry, France, 1993.

[14] R.E Moore, "Intervals Analysis" - Prentice Hall, 1966. 